\title{
FIXED POINTS OF NODAL CONTRACTIONS IN CONE METRIC SPACES
}

\author{
G. V. R. BABU AND G. N. ALEMAYEHU
}

\begin{abstract}
We introduce local power contractions and nodal contractions in cone metric spaces and prove the existence of fixed points of such contractions in cone metric spaces. Our theorems generalize the results of Haung and Zhang [L-G. Haung, X. Zhang, Cone metric spaces and fixed point theorems of contractive mappings, J. Math. Anal. Appl. 332 (2007) 1468-1476].
\end{abstract}

\section{Introduction}

In 2007, Haung and Zhang [2] generalized the concept of a metric space, replacing the set of real numbers by an ordered Banach space by defining the concept of a cone metric space which is more general than that of a metric space, and obtained fixed point theorems for mappings satisfying different contractive conditions.

Let $E$ be a real Banach space and $P$ be a subset of $E$. $P$ is called a cone if the following three conditions

(1) $P$ is closed, nonempty, and $P \neq\{0\}$,

(2) $a, b \in \mathbb{R}, a, b \geq 0, x, y \in P \Rightarrow a x+b y \in P$, and

(3) $x \in P$ and $-x \in P \Rightarrow x=0$.

Given a cone $P \subset E$, we define a partial order $\leq$ with respect to $P$ by $x \leq y$ if and only if $y-x \in P$. In this case we call $P$ an order cone. We write $x<y$ if $x \leq y$ and $x \neq y$; we write $x \ll y$ if $y-x \in$ int $P$, where int $P$ denotes the interior of $P$.

An order cone $P$ is called normal if there is a number $K>0$ such that for all $x, y \in E$,

$$
0 \leq x \leq y \text { implies }\|x\| \leq K\|y\| .
$$

Corresponding author: G. V. R. Babu.

Received January 5, 2010.

2000 Mathematics Subject Classification. 47H10, 54H25.

Key words and phrases. Cone metric spaces, nodal contractions, local power contractions, nodal points, fixed points 
Rezapour and Hamlbarani [3] observed that there is no normal cone with normal constant $K<1$.

Definition 1.1. Let $X$ be a nonempty set. If the mapping $d: X \times X \rightarrow E$ satisfies

(1) $0 \leq d(x, y)$ for all $x, y \in X$ and $d(x, y)=0$ if and only if $x=y$,

(2) $d(x, y)=d(y, x)$, for all $x, y \in X$, and

(3) $d(x, y) \leq d(x, z)+d(z, y)$, for all $x, y, z \in X$,

then $d$ is called a cone metric on $X$, and $(X, d)$ is called a cone metric space.

Definition 1.2. Let $(X, d)$ be a cone metric space and let $\left\{x_{n}\right\}$ be a sequence in $X$. We say that $\left\{x_{n}\right\}$ is

(1) a Cauchy sequence in $X$ if for each $c$ in $E$ with $0 \ll c$, there is an $N$ such that for all $m, n>$ $N, d\left(x_{m}, x_{n}\right) \ll c ;$

(2) a convergent sequence in $X$ if for each $c$ in $E$ with $0 \ll c$, there is $N$ such that for all $n>N$, $d\left(x_{n}, x\right) \ll c$ for some $x$ in $X$. In this case, we say that $\left\{x_{n}\right\}$ converges to $x$ in $X$ and we denote it by $\lim _{n \rightarrow \infty} x_{n}=x$ or $x_{n} \rightarrow x$ as $n \rightarrow \infty$.

We observe that if the cone is normal and the sequence $\left\{x_{n}\right\}$ is convergent, then the limit of $\left\{x_{n}\right\}$ is unique.

Lemma 1.3.(Haung and Zhang [2]). Let $(X, d)$ be a cone metric space, $P$ be a normal cone with normal constant $K$. Let $\left\{x_{n}\right\}$ be a sequence in $X$. Then

(i) $\left\{x_{n}\right\}$ converges to $x$ in $X$ if and only if $d\left(x_{n}, x\right) \rightarrow 0$ as $n \rightarrow \infty$,

(ii) $\left\{x_{n}\right\}$ Cauchy in $X$ if and only if $d\left(x_{m}, x_{n}\right) \rightarrow 0$ as $m, n \rightarrow \infty$.

Lemma 1.4.(Haung and Zhang [2]). Let $(X, d)$ be a cone metric space, $P$ be a normal cone with normal constant $K$. Let $\left\{x_{n}\right\}$ and $\left\{y_{n}\right\}$ be sequences in $X$ such that $x_{n} \rightarrow x$ and $y_{n} \rightarrow y$ as $n \rightarrow \infty$. Then $d\left(x_{n}, y_{n}\right) \rightarrow d(x, y)$ as $n \rightarrow \infty$.

The following is the 'Banach contraction principle in cone metric space'.

Theorem 1.5.(Haung and Zhang [2]). Let $(X, d)$ be a complete cone metric space, $P$ be a normal cone with normal constant $K$. Let $T: X \rightarrow X$ be a selfmap. Assume that $T$ is a contraction on $X$; i.e., there exists $\alpha \in[0,1)$ such that

$$
d(T x, T y) \leq \alpha d(x, y), \text { for all } x, y \in X .
$$


Then $T$ has a unique fixed point in $X$. Also, for any $x \in X$, the iterative sequence $\left\{T^{n} x\right\}$ converges to the fixed point.

A generalization of Banach contraction principle in cone metric spaces is the following theorem.

Theorem 1.6.(Haung and Zhang [2]). Let $(X, d)$ be a complete cone metric space, $P$ be a normal cone with normal constant $K$. Let $T: X \rightarrow X$ be a selfmap. Assume that there exist $\alpha \in[0,1)$ and a positive integer $n$ such that

$$
d\left(T^{n} x, T^{n} y\right) \leq \alpha d(x, y), \text { for all } x, y \in X
$$

Then $T$ has a unique fixed point in $X$.

Thoughout this paper we use the following notation: we denote the $n^{\text {th }}$ iterate of $T$ by $T^{n}$, and $T^{0}=I$, the identity mapping. For any $A \subset X, \operatorname{cl} A$ represents the closure of $A$; $\mathbb{R}$ denotes the set of all reals; and $\mathbb{N}$ denotes the set of all natural numbers.

Thoughout this paper we use the following notation: we denote the $n^{\text {th }}$ iterate of $T$ by $T^{n}$, and $T^{0}=I$, the identity mapping. For any $A \subset X, \operatorname{cl} A$ represents the closure of $A ; \mathbb{R}$ denotes the set of all reals; and $\mathbb{N}$ denotes the set of all natural numbers.

Definition 1.7.(Sehgal [4]). Let $(X, d)$ be a metric space and $T: X \rightarrow X$ be a selfmap. The mapping $T$ is called a local power contraction if there exists a constant $0 \leq \alpha<1$ and for each $x \in X$, there exists a positive integer $n=n(x)$ such that

$$
d\left(T^{n} x, T^{n} y\right) \leq \alpha d(x, y) \text { for all } y \text { in } X .
$$

In 1969, Sehgal [4] established that every continuous local power contraction on a complete metric space has a unique fixed point and the sequence of iterates of any point in the complete metric space converges to the fixed point. In 1970, Guseman [1] generalized it by relaxing the continuity assumption, and proved the following theorem.

Theorem 1.8.(Guseman [1]). Let $(X, d)$ be a complete metric space and $T: X \rightarrow X$ be a selfmap. Suppose there exists $B \subset X$ such that
(a) $T(B) \subset B$;
(b) $T$ is a local power contraction on $B$, and
(c) For some $x_{0} \in B, c l\left\{T^{n} x_{0}: n \geq 1\right\} \subset B$. 
Then $T$ has a unique fixed point $z$ in $B$ and $T^{n} x \rightarrow z$ as $n \rightarrow \infty$ for each $x \in B$. Furthermore, if $d\left(T^{n(z)} y, T^{n(z)} z\right) \leq \alpha d(y, z)$ for all $y \in X$, then $z$ is unique in $X$ and $T^{n} x_{0} \rightarrow z$ as $n \rightarrow \infty$ for each $x_{0} \in X$.

Also, Guseman [1] asserted the following.

Theorem 1.9. Let $(X, d)$ be a metric space, $T: X \rightarrow X$ be a mapping, and $u, y_{0} \in X$ with $T^{n} y_{0} \rightarrow$ $u$ as $n \rightarrow \infty$. If there exists a constant $0 \leq \alpha<1$ and there exists a positive integer $n=n(u)$ such that

$$
d\left(T^{n} x, T^{n} u\right) \leq \alpha d(x, u) \text { for all } x \in X .
$$

then $T$ has a unique fixed point $u$ in $X$ and $T^{n} x_{0} \rightarrow u$ as $n \rightarrow \infty$ for each $x_{0} \in X$.

In section 2 of this paper, we introduce local power contractions, nodal contractions, nodal points, and discuss the relation between nodal points and fixed points. In section 3 , we prove the existence of fixed points of local power contractions and establish the convergence of Picard iterations to the fixed point when the fixed point of the operator considered is unique (Theorem 3.2).

Also, we prove the existence of nodal points of nodal contractions in cone metric spaces (Theorem 3.3) and we apply this result in obtaining fixed points (Corollary 3.4). Our theorems generalize the results of Haung and Zhang [2].

\section{Local power contractions, nodal contractions and nodal points}

Definition 2.1. Let $(X, d)$ be a cone metric space with an order cone $P$ and $B$ be a nonempty subset of $X$. A mapping $T: B \rightarrow B$ is said to be a nodal contraction on $B$ if there exists a constant $\alpha \in[0,1)$, and there exists $b \in B$ and a positive integer $n=n(b)>1$ such that

$$
d\left(T^{n} x, T^{n} b\right) \leq \alpha d(x, b) \text { for all } x \text { in } B .
$$

In addition, if $T^{n} b=b$, the point $b$ is called a 'nodal point' of $T$.

If $n=1$ in (2.1.1), then we call $T$ a simple nodal contraction on $B$. In addition, if $T b=b$, then $b$ is called a simple nodal point of $T$.

Remark 2.2. (i) Every nodal contraction on a nonempty subset $B$ of $X$ need not be a simple nodal contraction on $B$; and

(ii) Every simple nodal point is a nodal point but its converse need not be true.

The following is an example for the justification of Remark 2.2. 
Example 2.3. Let $E=\mathbb{R}^{2}$, the Euclidean plane, and $P=\left\{(x, y) \in \mathbb{R}^{2}: x \geq 0, y \geq 0\right\}$. Then $P$ is a cone in $E$. Let $X=\left\{(x, 0) \in \mathbb{R}^{2}: 0 \leq x \leq 1\right\} \cup\left\{(0, x) \in \mathbb{R}^{2}: 0 \leq x \leq 1\right\}$.

The mapping $d: X \times X \rightarrow E$ defined by

$$
d((x, 0),(y, 0))=d((0, x),(0, y))=(|x-y|,|x-y|)
$$

and

$$
d((x, 0),(0, y))=d((0, y),(x, 0))=(x+y, x+y)
$$

is a cone metric on $X$.

We define $T: X \rightarrow X$ by $T((x, 0))=(0, x)$ and $T((0, x))=\left(\frac{1}{2} x, 0\right)$.

We take $b=(1,0)$ in $X$. Then

(i) $d\left(T^{2}((x, 0)), T^{2}((1,0))\right)=d\left(\left(\frac{1}{2} x, 0\right),\left(\frac{1}{2}, 0\right)\right)=\frac{1}{2}(|x-1|,|x-1|)=\frac{1}{2} d((x, 0),(1,0))$, and

(ii) $d\left(T^{2}((0, y)), T^{2}((1,0))\right)=d\left(\left(0, \frac{1}{2} y\right),\left(\frac{1}{2}, 0\right)\right)=\frac{1}{2}(y+1, y+1)=\frac{1}{2} d((0, y),(1,0))$.

Hence, $T$ is a nodal contraction on $X$ with $n=2$ and $\alpha=\frac{1}{2}$. Here we observe that $T$ is not a simple nodal contraction on $X$; since, for any $\alpha \in[0,1)$ and for any $(x, 0) \in X$,

$$
d(T((x, 0)), T((1,0))=(1-x, 1-x) \not \leq \alpha(1-x, 1-x)=\alpha d((x, 0),(1,0)) .
$$

Also, we observe that the point $(0,0)$ is a nodal point of $T$ in $X$, which is not a simple nodal point of $T$ in $X$.

Now, the following question is natural:

"Is every nodal contraction has a fixed point?"

The answer to this question is not affirmative, which is shown by the following example.

Example 2.4. Let $E=\mathbb{R}^{2}$, the Euclidean plane, and $P=\left\{(x, y) \in \mathbb{R}^{2}: x \geq 0, y \geq 0\right\}$. Then $P$ is a cone in $E$. Let $X=\left\{(x, 0) \in \mathbb{R}^{2}: 0<x \leq 1\right\} \cup\left\{(0, x) \in \mathbb{R}^{2}: 0<x \leq 1\right\}$.

The mapping $d: X \times X \rightarrow E$ defined by

$$
d((x, 0),(y, 0))=d((0, x),(0, y))=(|x-y|,|x-y|)
$$

and

$$
d((x, 0),(0, y))=d((0, y),(x, 0))=(x+y, x+y)
$$

is a cone metric on $X$. We define $T: X \rightarrow X$ by

$$
T((x, 0))= \begin{cases}(0, x) & \text { if } x \text { is rational } \\ \left(0, \frac{1}{2} x\right) & \text { if } x \text { is irrational, }\end{cases}
$$


and

$$
T((0, x))= \begin{cases}\left(\frac{1}{2} x, 0\right) & \text { if } x \text { is rational } \\ (x, 0) & \text { if } x \text { is irrational. }\end{cases}
$$

Then $T$ is a nodal contraction on $X$ with $n=2$ and $\alpha=\frac{1}{2}$. We observe that $T$ has no fixed points in $X$. Thus we have the following question.

Question 1. What additional hypotheses is required for a nodal contraction to have a fixed point?

We answer this question in Proposition 2.10 and use it to prove our main results of Section 3.

Remark 2.5. The following example shows that every simple nodal contraction need not have a simple nodal point.

Example 2.6. Let $E=\mathbb{R}$, and $P=\{x \in \mathbb{R}: x \geq 0\}$, a cone of real line. Let $X=[0,1] \times[0,1]$. We define the cone metric $d$ on $X$ as the usual metric on $\mathbb{R}^{2}$. We define $T: X \rightarrow X$ by

$$
T((x, y))= \begin{cases}\left(\frac{1}{2} x, \frac{1}{2} y+\frac{1}{2}\right) & \text { if }(x, y) \in X-\{(0,1)\} \\ (0,0) & \text { if }(x, y)=(0,1) .\end{cases}
$$

We choose $b=(0,0) \in X$. Then $d(T((x, y)), T((0,0)))=\frac{1}{2} d((x, y),(0,0))$ for all $(x, y) \in X$. Hence, $T$ is a simple nodal contraction on $X$. But $T$ has no simple nodal points in $X$.

Here we observe that Example 2.6 does not contradict Theorem 1.5, since $T$ is not a contraction; for, the points $(1,1),(0,1) \in X$, we have $d(T((1,1)), T((0,1)))=\frac{\sqrt{5}}{2} \not \leq \alpha d((1,1),(0,1))$ for any $0 \leq \alpha<1$.

Question 2. Under what hypotheses, a simple nodal contraction has a fixed point?

We answer this question in Corollary 3.5.

Definition 2.7. Let $(X, d)$ be a cone metric space with an ordered cone $P$. We say that $T: X \rightarrow$ $X$ has

(1) a local simple nodal point if there exists a subset $B$ of $X$ such that $T$ has a simple nodal point in $B$;

(2) a global simple nodal point if $T$ has a simple nodal point in $X$.

Remark 2.8. Every local simple nodal point need not be a global simple nodal point. 
Example 2.9. Let $E, P, X$ and $d$ be as given in Example 2.3. We define $T: X \rightarrow X$ by

$$
T((x, 0))= \begin{cases}(0,0) & \text { if } x \text { is irrational } \\ (0, x) & \text { if } x \text { is rational, }\end{cases}
$$

and

$$
T((0, x))= \begin{cases}(0,0) & \text { if } x \text { is irrational } \\ (x, 0) & \text { if } x \text { is rational. }\end{cases}
$$

Let $B=\{(0,0)\} \cup\{(x, 0) \in X: x$ is irrational $\} \cup\{(0, x) \in X: x$ is irrational $\}$. Then $T$ is a simple nodal contraction on $B$ and the point $(0,0)$ is a local simple nodal point of $T$ in $B$.

We observe that $T$ has no global simple nodal points, for $d(T((x, 0)), T((y, 0)))=d((x, 0),(y, 0))$ for all $(x, 0),(y, 0) \in A$, where $A=\{(x, 0) \in X: x$ is rational $\} \cup\{(0, x) \in X: x$ is rational $\}$.

Proposition 2.10. Let $(X, d)$ be a cone metric space with an order cone $P$. Let $T: X \rightarrow X$ be a selfmap. Then every nodal point of $T$ in a nonempty subset $B$ of $X$ is a unique fixed point of $T$ in $B$.

Proof. Let $z \in B$ be a nodal point of $T$. Then there exists a constant $0 \leq \alpha<1$ and a positive integer $n=n(z)>1$ such that $T^{n} z=z$ and

$$
d\left(T^{n} x, T^{n} z\right) \leq \alpha d(x, z) \text { for all } x \in B
$$

From the inequality (2.10.1), $z$ is the unique fixed point of $T^{n}$ in $B$.

Now $T z=T\left(T^{n} z\right)=T^{n}(T z)$. Then, by the uniqueness of the fixed point of $T^{n}, T z=z$. If $T w=w$ for some $w \in B$, it follows that $T^{n} w=w$ and hence $w=z$. Hence, $z$ is the unique fixed point of $T$ in $B$.

Definition 2.11. Let $(X, d)$ be a cone metric space with an order cone $P$. Let $B \subseteq X$. A mapping $T: B \rightarrow B$ is said to be a local power contraction on $B$ if there exists a constant $0 \leq \alpha<1$ and for each $y \in X$, there exists a positive integer $n=n(y)$ such that

$$
d\left(T^{n} x, T^{n} y\right) \leq \alpha d(x, y) \text { for all } x \text { in } B .
$$

Here, we call $\alpha$ a local power contraction constant of $T$ in $B$.

Remark 2.12. From the Definition 2.11, it is easy to see that every local power contraction is a nodal contraction if $n>1$; and every local power contraction is a simple nodal contraction if $n=1$. The following examples show that its converse need not be true even when the cone is a nonnegative real line. 
Example 2.13. Let $E=\mathbb{R}$, and $P=\{x \in \mathbb{R}: x \geq 0\}$. Let $X=[0,1] \times[0,1]$. We define the cone metric $d$ on $X$ as the usual metric on $\mathbb{R}^{2}$. We define $T: X \rightarrow X$ by

$$
T((x, y))= \begin{cases}\left(\frac{1}{2} y+\frac{1}{2}, x\right) & \text { if }(x, y) \in X-\{(0,1),(1,0)\} \\ (1,0) & \text { if }(x, y)=(0,1) \\ (0,1) & \text { if }(x, y)=(1,0) .\end{cases}
$$

We choose $b=(0,0) \in X$. If $(x, y) \in X-\{(0,1),(1,0)\}$, then $d\left(T^{2}((x, y)), T^{2}((0,0))\right)=\frac{1}{2} d((x, y),(0,0)) \leq \frac{1}{\sqrt{2}} d((x, y),(0,0))$, and if $(x, y) \in\{(0,1),(1,0)\}$, then $d\left(T^{2}((x, y)), T^{2}((0,0))\right)=\frac{1}{\sqrt{2}} d((x, y),(0,0))$. Hence, $T$ is a nodal contraction on $X$ with $n=2$ and $\alpha=\frac{1}{\sqrt{2}}$.

Here, we observe that $T$ is not a local power contraction on $X$; for, the points $(1,1),(1,0) \in$ $X$, we have $d\left(T^{n}((1,1)), T^{n}((1,0))\right)=1 \not \leq \alpha d((1,1),(1,0))$ for any $0 \leq \alpha<1$ and $n=1,2,3, \ldots$.

Example 2.14. Let $E, P, X$, and $d$ be as given in Example. We define $T: X \rightarrow X$ by

$$
T((x, y))= \begin{cases}\left(\frac{1}{2} x, \frac{1}{2} y+\frac{1}{2}\right) & \text { if }(x, y) \in X-\left\{\left(1, \frac{3}{4}\right),(1,1)\right\} \\ \left(1, \frac{3}{4}\right) & \text { if }(x, y)=(1,1) \\ (1,1) & \text { if }(x, y)=\left(1, \frac{3}{4}\right)\end{cases}
$$

We choose $b=(0,0) \in X$. If $(x, y) \in X-\left\{\left(1, \frac{3}{4}\right),(1,1)\right\}$, then

and

$$
\begin{aligned}
& d(T((x, y)), T((0,0)))=\frac{1}{2} d((x, y),(0,0)) \leq \frac{2}{\sqrt{5}} d((x, y),(0,0)), \\
& d(T((1,1)), T((0,0)))=\frac{\sqrt{17}}{4}=\frac{\sqrt{17}}{4 \sqrt{2}} d((1,1),(0,0)) \leq \frac{2}{\sqrt{5}} d((1,1),(0,0)),
\end{aligned}
$$

$$
d\left(T\left(\left(1, \frac{3}{4}\right)\right), T((0,0))\right)=\frac{\sqrt{5}}{2}=\frac{2}{\sqrt{5}} d\left(\left(1, \frac{3}{4}\right),(0,0)\right) .
$$

Hence, $T$ is a simple nodal contraction on $X$ with $\alpha=\frac{2}{\sqrt{5}}$.

But $T$ is not a local power contraction on $X$; for, the points $(1,1),(0,1) \in X$,

$$
d\left(T^{n}((1,1)), T^{n}((0,1))\right)=\left\{\begin{array}{ll}
1 & \text { if } n \text { is even } \\
\frac{\sqrt{17}}{4} & \text { if } n \text { is odd }
\end{array}\right. \text { so that }
$$

$d\left(T^{n}((1,1)), T^{n}((0,1))\right) \not \leq \alpha d((1,1),(0,1))$ for any $0 \leq \alpha<1$ and $n=1,2,3, \ldots$ 


\section{Main results}

Proposition 3.1. Let $(X, d)$ be a cone metric space, $P$ be a normal cone with normal constant $K$. Let $T: X \rightarrow X$ be a selfmap. Then every nodal point of $T$ in a nonempty subset $B$ of $X$ with $T(B) \subset B$ is a unique fixed point of $T$ in $B$ and $T^{n}(x)$ converges to the unique fixed point for each $x$ in $B$.

\section{Proof.}

Let $z \in B$ be a nodal point of a map $T$. Then there exists a constant $0 \leq \alpha<1$ and a positive integer $n=n(z)>1$ such that $T^{n} z=z$ and

$$
d\left(T^{n} x, T^{n} z\right) \leq \alpha d(x, z) \text { for all } x \in B
$$

By Proposition 2.10, $z$ is a unique fixed point of $T$ in $B$. Now, let $y_{0} \in B$. Since $T(B) \subset B$, we have $\left\{T^{m} y_{0}: m \geq 1\right\} \subset B$. Let $\delta\left(y_{0}\right)=\max \left\{\left\|d\left(T^{m} y_{0}, z\right)\right\|: m=1,2, \ldots, n-1\right.$. $\}$. Let $m \in \mathbb{N} \cup\{0\}$. For $m \geq n$, we write $m=r n+s$ where $r$ is a nonnegative integer and $s \in\{0,1,2, \ldots, n-1\}$.

Then,

$$
d\left(T^{m} y_{0}, z\right)=d\left(T^{r n+s}\left(y_{0}\right), T^{n} z\right) \leq \alpha d\left(T^{(r-1) n+s}\left(y_{0}\right), z\right) \leq \cdots \leq \alpha^{r} d\left(T^{s}\left(y_{0}\right), z\right) .
$$

Hence,

$$
d\left(T^{m} y_{0}, z\right) \leq \alpha^{r} d\left(T^{s}\left(y_{0}\right), z\right)
$$

From (3.1.2), by the normality of the cone $P$ with normal constant $K$, we have

$$
\left\|d\left(T^{m} y_{0}, z\right)\right\| \leq K \alpha^{r}\left\|d\left(T^{s}\left(y_{0}\right), z\right)\right\| \leq K \alpha^{r} \delta\left(y_{0}\right) \rightarrow 0 \text { as } m \rightarrow \infty
$$

Hence, $T^{m}(x) \rightarrow z$ as $m \rightarrow \infty$ for each $x \in B$.

Theorem 3.2. Let $(X, d)$ be a complete cone metric space, $P$ be a normal cone with normal constant $K$. Let $T: X \rightarrow X$ be a selfmap. Suppose that there exists $B \subset X$ such that

(a) $T(B) \subset B$;

(b) $T$ is a local power contraction on B with local power contraction constant $\alpha$; and

(c) For some $x_{0} \in B, c l\left\{T^{n} x_{0}: n \geq 1\right\} \subset B$.

Then there exists a unique $z \in B$ such that $T z=z$ and $T^{n}(y) \rightarrow z$ as $n \rightarrow \infty$ for each $y \in B$. Further, if $z$ is a nodal point of $T$ in $X$, then $z$ is unique in $X$ and $T^{n}(x) \rightarrow z$ as $n \rightarrow \infty$ for each $x \in X$. 
Proof. Let $y \in B$. We write $\gamma(y)=\sup _{n}\left\|d\left(T^{n} y, y\right)\right\|$. First we show that $\gamma(y)<\infty$. since $y \in B$ and $T^{n}(B) \subset B$ for each $n=1,2,3, \ldots$, and Since $T$ is a local power contraction on $B$, there exists a positive integer $m=m(y)$ such that

$$
d\left(T^{m} T^{n} y, T^{m} y\right) \leq \alpha d\left(T^{n} y, y\right) \text { for all } x \in B .
$$

Let $n \in \mathbb{N} \cup\{0\}$ and let $n \geq m$, we write $n=r m+s$, where $r$ is a nonnegative integer and $s \in\{0,1,2, \ldots, m-1\}$. We write $\delta(y)=\max \left\{\left\|d\left(T^{l} y, y\right)\right\|: l=1,2, \ldots, m.\right\}<\infty$. Now

$$
\begin{aligned}
d\left(T^{n} y, y\right) & \leq d\left(T^{r+s} y, T^{m} y\right)+d\left(T^{m} y, y\right) \\
& \leq \alpha d\left(T^{(r-1) m+s} y, y\right)+d\left(T^{m} y, y\right) \\
& \leq \alpha^{2} d\left(T^{(r-2) m+s} y, y\right)+(\alpha+1) d\left(T^{m} y, y\right) \\
& \vdots \\
& \leq \alpha^{r} d\left(T^{s} y, y\right)+\left(\alpha^{r-1}+\alpha^{r-2}+\cdots+\alpha+1\right) d\left(T^{m} y, y\right) .
\end{aligned}
$$

From (3.2.1) and by the normality of the cone $P$, we have

$$
\begin{aligned}
\left\|d\left(T^{n} y, y\right)\right\| & \leq K\left\|\alpha^{r} d\left(T^{s} y, y\right)+\left(\alpha^{r-1}+\alpha^{r-2}+\cdots+\alpha+1\right) d\left(T^{m} y, y\right)\right\| \\
& \leq K\left(\alpha^{r}\left\|d\left(T^{s} y, y\right)\right\|+\left(\alpha^{r-1}+\alpha^{r-2}+\cdots+\alpha+1\right)\left\|d\left(T^{m} y, y\right)\right\|\right) \\
& \leq K\left(\alpha^{r}+\alpha^{r-2}+\cdots+\alpha+1\right) \delta(y) \\
& \leq \frac{K}{1-\alpha} \delta(y),
\end{aligned}
$$

and hence it follows that

$$
\sup _{n}\left\|d\left(T^{n} y, y\right)\right\| \leq \frac{K}{1-\alpha} \delta(y)<\infty .
$$

This shows that $\gamma(y)<\infty$.

Let $x_{0} \in B$ as hypothesized in (c). Then by (b), there exists a positive integer $m_{0}=m\left(x_{0}\right)$ such that

$$
d\left(T^{m_{0}} y, T^{m_{0}} x_{0}\right) \leq \alpha d\left(y, x_{0}\right) \text { for all } y \in B .
$$

Write $x_{1}=T^{m_{0}}\left(x_{0}\right)$. Then by (a), $x_{1} \in B$. Again, by (b), there exists a positive integer $m_{1}=m\left(x_{1}\right)$ such that

$$
d\left(T^{m_{1}} y, T^{m_{1}} x_{1}\right) \leq \alpha d\left(y, x_{1}\right) \text { for all } y \in B .
$$

Write $x_{2}=T^{m_{1}}\left(x_{1}\right)$. Then by (a), $x_{2} \in B$.

On continuing this process, we obtain a sequence of positive integers $\left\{m_{j}\right\}$ where $m_{j}=$ $m\left(x_{j}\right)$ for all $j$ and a sequence $\left\{x_{j}\right\}$ defined by $x_{j+1}=T^{m_{j}} x_{j}, j=0,1,2, \ldots$. Since

$$
d\left(x_{j+1}, x_{j}\right) \leq d\left(T^{m_{j}} x_{j}, T^{m_{j-1}} x_{j-1}\right)=d\left(T^{m_{j}} T^{m_{j-1}} x_{j-1}, T^{m_{j-1}} x_{j-1}\right)
$$




$$
\begin{aligned}
& \leq \alpha d\left(T^{m_{j}} x_{j-1}, x_{j-1}\right) \\
& \vdots \\
& \leq \alpha^{j} d\left(T^{m_{j}} x_{0}, x_{0}\right), j=0,1,2, \ldots,
\end{aligned}
$$

we have

$$
d\left(x_{j}, x_{i}\right) \leq \alpha^{j-1} d\left(T^{m_{j-1}} x_{0}, x_{0}\right)+\alpha^{j-2} d\left(T^{m_{j-2}} x_{0}, x_{0}\right)+\cdots+\alpha^{i} d\left(T^{m_{i}} x_{0}, x_{0}\right) \text { for all } j>i
$$

Now, using the normality of $P$, we have

$$
\left\|d\left(x_{j}, x_{i}\right)\right\| \leq \frac{K \alpha^{i}}{1-\alpha} \gamma\left(x_{0}\right) \rightarrow 0 \text { as } i \rightarrow \infty .
$$

Hence, the sequence $\left\{x_{j}\right\}$ is Cauchy in $B$.

By the completeness of $X$ and (c), we have $x_{j} \rightarrow z \in B$ as $j \rightarrow \infty$. Hence, by (b), there exists a positive integer $n=n(z)$ such that

$$
d\left(T^{n} y, T^{n} z\right) \leq \alpha d(y, z) \text { for each } y \in B .
$$

In particular, for $y=x_{j}$, we have

$$
d\left(T^{n} x_{j}, T^{n} z\right) \leq \alpha d\left(x_{j}, z\right), j=1,2,3, \ldots
$$

Now, using the normality of $P$, we have

$$
\left\|d\left(T^{n} x_{j}, T^{n} z\right)\right\| \leq K \alpha\left\|d\left(x_{j}, z\right)\right\| \rightarrow 0 \text { as } j \rightarrow \infty .
$$

Hence, $T^{n} x_{j} \rightarrow T^{n} z$ as $j \rightarrow \infty$. Now

$$
d\left(T^{n} x_{j}, x_{j}\right) \leq \alpha d\left(T^{n} x_{j-1}, x_{j-1}\right) \leq \cdots \leq \alpha^{j} d\left(T^{n} x_{0}, x_{0}\right), j=1,2,3, \ldots
$$

Hence,

$$
\left\|d\left(T^{n} x_{j}, x_{j}\right)\right\| \leq K \alpha^{j}\left\|d\left(T^{n} x_{0}, x_{0}\right)\right\| \rightarrow 0 \text { as } j \rightarrow \infty .
$$

Thus,

$$
d\left(T^{n} x_{j}, x_{j}\right) \rightarrow 0 \text { as } j \rightarrow \infty .
$$

Now, by Lemma 1.4, it follows that $T^{n} z=z$. Hence, $z$ is the unique nodal point of $T$ in $B$. By Proposition 3.1, $z$ is the unique fixed point of $T$ in $B$ and $T^{n} y \rightarrow z$ as $n \rightarrow \infty$, for each $y \in B$. The last assertion of Theorem 3.2 follows directly from Proposition??

This completes the proof of the theorem. 
Theorem 3.3. Let $(X, d)$ be a cone metric space, $P$ be a normal cone with normal constant $K$. Let $T: X \rightarrow X$ be a selfmap. Suppose that there exist $z, x_{0} \in X$ with $T^{m} x_{0} \rightarrow z$ as $m \rightarrow \infty$. If there exists a constant $0 \leq \alpha<1$ and there exists a positive integer $n=n(z)$ such that

$$
d\left(T^{n} x, T^{n} z\right) \leq \alpha d(x, z) \text { for all } x \in X
$$

then $z$ is a nodal point of $T$ in $X$.

Proof. Since $T^{m} x_{0} \rightarrow z$ as $m \rightarrow \infty$, then $T^{n+m} x_{0} \rightarrow z$ as $m \rightarrow \infty$. Now

$$
\begin{aligned}
d\left(T^{n} z, z\right) & \leq d\left(T^{n} z, T^{n+m} x_{0}\right)+d\left(T^{n+m} x_{0}, T^{m} x_{0}\right)+d\left(T^{m} x_{0}, z\right) \\
& \leq(1+\alpha) d\left(z, T^{m} x_{0}\right)+d\left(T^{n+m} x_{0}, T^{m} x_{0}\right)
\end{aligned}
$$

From (3.3.2), by the normality of the cone $P$ and Lemma 1.4, we have

$$
\left\|d\left(T^{n} z, z\right)\right\| \leq K\left[(1+\alpha)\left\|d\left(z, T^{m} x_{0}\right)\right\|+\left\|d\left(T^{n+m} x_{0}, T^{m} x_{0}\right)\right\|\right] \rightarrow 0 \text { as } m \rightarrow \infty .
$$

Hence, $T^{n} z=z$.

Hence, the theorem follows.

Corollary 3.4. Let $(X, d)$ be a cone metric space, $P$ be a normal cone with normal constant $K$. Let $T: X \rightarrow X$ be a selfmap satisfying the hypotheses of Theorem 3.3. Then $z$ is a fixed point of $T$ in $X$ and $T^{n} x \rightarrow z$ as $n \rightarrow \infty$ for each $x \in X$.

Proof. By Theorem 3.3, $z \in X$ is a nodal point of $T$ and hence, by Proposition 3.1, the conclusion of the theorem follows.

Corollary 3.5. Let $(X, d)$ be a cone metric space, $P$ be a normal cone with normal constant $K$. Let $T: X \rightarrow X$ be a selfmap. Suppose that there exist $z, x_{0} \in X$ with $T^{n} x_{0} \rightarrow z$ as $n \rightarrow \infty$. If there exists a constant $0 \leq \alpha<1$ such that

$$
d(T x, T z) \leq \alpha d(x, z) \text { for all } x \in X
$$

then $z$ is a fixed point of $T$ in $X$; in other words, $z$ is a simple nodal point of $T$ in $X$. Also, $T^{n} x \rightarrow z$ as $n \rightarrow \infty$ for each $x \in X$.

Proof. Follows from Corollary 3.4 with $n=1$ in (3.3.1).

Remark 3.6. Theorem 3.2 and Corollary 3.4 are extensions of Theorem 1.8 and Theorem 1.9 to cone metric spaces respectively. 
Example 3.7. Let $E, P, X, d, T$ and $B$ be given as in the Example 2.9. Then $P$ is a normal cone in $E$ and $T(B) \subset B$. For any $\alpha \in[0,1), T$ satisfies the contraction condition

$$
d\left(T\left(\left(x_{1}, x_{2}\right)\right), T\left(\left(y_{1}, y_{2}\right)\right)\right) \leq \alpha d\left(\left(x_{1}, x_{2}\right),\left(y_{1}, y_{2}\right)\right) \text { for all }\left(x_{1}, x_{2}\right),\left(y_{1}, y_{2}\right) \in B,
$$

so that $T$ is a local power contraction on $B$. Also, condition (c) of Theorem 3.2 trivially holds on $B$. Hence, $T$ satisfies all the conditions of Theorem 3.2 and the point $(0,0)$ is the unique fixed point of $T$ in $X$.

But, for $(1,0),(0,1) \in X$, we have $d(T((1,0)), T((0,1)))=d((1,0),(0,1))$ so that $T$ is not a contraction on the cone metric space $X$. Thus, Theorem 1.5 and Theorem 1.6 are not applicable. This shows that Theorem 3.2 generalizes Theorem 1.5 and Theorem 1.6, which in turn Corollary 3.4 is also a generalization of Theorem 1.6.

Example 3.8. Let $E, P, X, d$ and $T$ be given as in the Example 2.3. Then $P$ is a normal cone in $E$. We have $T^{n}((0,1))=\left(0, \frac{1}{2^{m}}\right)$ if $n=2 m$ and $T^{n}((0,1))=\left(\frac{1}{2^{m+1}}, 0\right)$ if $n=2 m+1, m=0,1,2, \ldots$ so that $T^{n}((0,1)) \rightarrow(0,0)$ as $n \rightarrow \infty$. Also, $d\left(T^{2}((x, y)), T^{2}((0,0))\right) \leq \frac{1}{2} d((x, y),(0,0))$, for all $(x, y) \in X$, so that $T^{2}$ satisfies all the conditions of Corollary 3.4 and $T$ has a unique fixed point $(0,0)$ in $X$.

\section{References}

[1] L. F. Guseman, JR., Fixed point theorems for mappings with a contractive iterate at a point, Proc. Amer. Math. Soc., 26(1970), 615-618.

[2] L-G. Haung and X. Zhang, Cone metric spaces and fixed point theorems of contractive mappings, J. Math. Anal. Appl., 332(2007), 1468-1476.

[3] Sh. Rezapour and R. Hamlbarani, Some notes on the paper "Cone metric spaces and fixed point theorems of contractive mappings", J. Math. Anal. Appl., 345 (2008), 719-724.

[4] V. M. Sehgal, A fixed point theorem for mappings with a contractive iterate at a point, Proc. Amer. Math. Soc., 23(1969), 631-634.

Department of Mathematics, Andhra University, Visakhapatnam-530 003, Andhra Pradesh, India.

E-mail: gvr_babu@hotmail.com

Department of Mathematics, Jimma University, Jimma, P.O.Box 378, Ethiopia.

Department of Mathematics, Andhra University, Visakhapatnam-530 003, Andhra Pradesh, India.

E-mail: alemg1972@gmail.com 